\title{
BIM PROCESS, ONTOLOGIES AND INTERCHANGE PLATFORM FOR CULTURAL ARCHITECTURAL HERITAGE MANAGEMENT: STATE OF ART AND DEVELOPMENT PERSPECTIVES
}

\author{
A. Pili \\ Politecnico di Milano, Department of architecture, built environment and construction engineering
}

\begin{abstract}
KEY WORDS: Ontologies, IFC for $\mathrm{CH}$, Interoperability, Building Information Modelling, Conservation process, Process
\end{abstract} management, Interchange standards, $\mathrm{CDE}$

\begin{abstract}
:
Ontology meaning and its declination in computer science are defined in the contribution. Some experiences of using ontologies for Cultural Heritage frame the state of the art. Specific ontologies for the conservation process allows the definition of classes and their description through attributes. Subjects, means, tools, relationships, and planning of activities, times and costs are included in the process. Ontologies so defined are also the basis for the definition of an interoperability protocol for Cultural Heritage. Interoperability means exchange among the tools, with the guarantee that data and meanings transmitted are correctly interpreted by the receiving system. The paper proposes the kind of informative model for built heritage. Defining the basis for ontologies is the goal. The flow of information in a Common Data Environment, a data exchange platform, is necessary to ensure proper data and process management.
\end{abstract}

\section{INTRODUCTION}

The proposed contribution come from Ph.D project which I am conducing, according to CHERIE Project activities. CHERIE (Cultural Heritage Interoperable Environment) Project is developed by Politecnico di Milano, University of Genoa, La Sapienza University of Rome, University of Naple Federico II, University of Suor Orsola Benincasa, Stress S.c.a.r.l. Both Ph.D and CHERIE activities are still ongoing.

Starting from January 2019 the BIM use is mandatary for public procurements. Also, the works on the public cultural heritage are subject to this obligation. Because of their programming for standardized processes, BIM tools on the market are not adequate to respond to regulatory requirements. Even the legislation concerning digital management of information processes is lacking on the issues of cultural value and on the management processes of the existing architectural heritage.

A historical cultural building is a complex system of specificities, relationships and processes. A twofold temporal aspect characterizes the historic buildings. Unlike a new building, a historic building presents a past life. On the time line, a new building assumes a positive trend starting from a "condition 0" with the requirement detection phase and continuing according to its life cycle. A historic building, on the other hand, begins its positive path towards management with a substantial store of information.

The elapsed time implies the presence of specific nonrepeatable characters. These characters are the result of the combination of many variables. Manual production method, historical period and architectural style, level of empirical and technological knowledge, location and availability of raw materials, local culture, stratification of the interventions and so on are some of these variables. This specificity translates into unique objects that cannot be standardized. Because of the heterogeneity of values, the information modelling tools currently on the market are not able to return a model capable of accepting, describing, synthesizing and correctly transmitting the large amount of information. Depending on the manual production, the shape and geometric characteristics are also difficult to represent by means of BIM.

\section{ONTOLOGY: FROM PHILOSOPHY TO COMPUTER SCIENCE}

\subsection{Ontology concept}

Ontology concept derive from classical philosophy. It literally means "study on being". In the Aristotelian view being as such is determined by multiple attributes that contribute to its singularity. Categories describe the predicates of being, its properties and relationships. The concept of ontology and categories has been taken up and elaborated by several philosophers over time. Although the concept has theoretical origins, ontology has also been used in typically practical disciplines such as medicine, physics and computer science. Information technology exploits the ability of ontology to conceptualize, describe and represent the entities within a domain and the relationships between them. What "exists" can be represented. As in philosophy, computer ontologies describe entities in relation to their capacity for change. Therefore, ontology is an explicit specification of a conceptualization. (Gruber, 1992) In operational terms, an ontology defines a common vocabulary for sharing information within a domain. (Noy and McGuinness, 2000) Ontologies allow to describe and conceptualize a complex system and to share information without error of interpretation.

\subsection{Ontology versus database}

Relational databases are also used to manage informational data, but some substantial differences existing between two methods.

The first one, "Unique Name Assumption", is an important term of comparison. It means that different names corresponding to different entities. OWL (Ontology Web Language) does not use 
this assumption but allows the recognition of multiple names for the same entity.

The use of CWA (Close World Assumption) or OWA (Open World Assumption) is another difference between ontologies and databases. The CWA system considers the assumption that if a thing is true it is recognized as such, if it is not true it is false. Opposite, the OWA considers that the lack of knowledge of an information does not imply the untrue. Databases generally use CWA, while for many knowledge bases and semantic webs, OWA is used (Razniewski, et al., 2016. Reiter, 1977) In practical terms, missing information in a database returns the value " 0 ", while in ontology the lack of a data is considered an "unknown" value. (Sir et al., 2015)

Other features are different between two methods, such as how to compile or the dependency of properties. There is several literatures on relational database, all these proposal have marked the lacks of relational database about semantic representation. In opposite, literature about ontology underline the semantic enrichment of data. However, there are also some similarities. The user's needs and purpose define the choice. (Martinez-Cruz, et al., 2011)

In the proposed case, the aim is to create standards for the interoperability of information models, which means making information models able to exchange and understand data and information.

\subsection{Definition of IFC standard}

Interoperability is the ability of a system to be correctly understood when it interfaces with another system.

If two systems have syntactic interoperability, they can communicate with each other and exchange information. Instead, semantic interoperability allows the receiving system to automatically interpret information. In this case, the content of the information is unambiguously defined: what has been transmitted is the same as what is understood.

Semantic interoperability concerns not only the transfer of data (syntax) but the transmission of their meaning (semantics). (Bittner et al, 2006) The completeness of syntax and semantics, data and information, is obtained by linking each element with a shared vocabulary. Vocabulary related to ontologies provides the ability to interpret meaning. As explained above, ontologies provide the ability to associate multiple names with the same entity. An ontology defines the words and concepts (meanings) used to describe and represent an area of knowledge. (Obrst, 2003) This means connecting the meaning of familiar words to unknown objects.

Computer systems work with entities with different degrees of specificity, from general to detail. (Kupcík, et al., 2012) Working in levels of detail means using a hierarchy. A hierarchical ontology is represented in not cyclical graph form in which a node defines a concept. Hierarchical ontology classifies concepts at each level and proceeds from general to detail (Khan and Safyan, 2014) according to father-son logic.

Interoperability implies the possibility of exchanging information both among similar products and among different versions of the same product. In order to guarantee interoperability, the definition of open standards is necessary. Open standards are available to create new products that can correctly transmit and receive information. In the absence of open standards every software should translate the data according to its own semantics (Laakso and Kiviniemi, 2012) with real possibility loss of information.

In the architecture, engineering and construction (AEC) industry different domains perform different tasks, depending on the purpose the models are different. It is important to remember the concept that "a model represents reality for the given purpose, the model is an abstraction of reality in the sense that it cannot represent all aspects of reality" as stated by Rothenberg. (Rothenberg, 1989) Essentially, this concept says that reality cannot be represented by a single model, therefore a single model cannot exist. It means that, in the AEC more models to represent reality exists and therefore more BIM are needed. To think that BIM is a unique model in which everyone works on the same data is an error. (Gholami, et al., 2015)

This concept also applies to the built heritage, where in addition to the already recognized information models (architectural, structural, systems and so on), a model that represents historical-cultural values is also present. The presence of several models with different purpose for each one means that some singularities that may not be relevant to an aspect may instead be essential for others. (Della Torre et al., 2017)

IFC (Industry Foundation Classes) is open format protocol that describes the data relating to the construction industry. It is registered by ISO 16739-1: 2018 (Industry Foundation Classes (IFC) for data sharing in the construction and facility management industries - Part 1: Scheme data) (ISO 16739-1: 2018). Its first version was developed by the Industry Alliance for Interoperability, later called the International Alliance for Interoperability and today buildingSMART International, which continues its development activity. Ensuring interoperability in architecture, engineering and construction (AEC) and facility management (FM) processes using informative models is the constant goal of buildingSMART International. According to the premises described so far, ontologies present the correct way to identify and describe the properties of objects in a sharing and interoperability environment. (Lee et al., 2008)

IFC defines an entity relationship model based on EXPRESS. It is composed of several hundred entities organized in an objectbased hierarchy of inheritance. EXPRESS is a standard of data modelling language standardized by ISO 10303-11: 2004/2008 (ISO 10303-11:2004). It is used to identify classes, class attributes and relationships among them. EXPRESS is not a programming language, it is a data specification language. EXPRESS-G is its graphic notation standard, that is graphic representation. EXPRESS-G allows to view the structure of the data model in a more understandable way.

\section{THE STATE OF THE ART}

\subsection{Ontologies for Cultural Heritage}

In the field of cultural heritage, attempts to digitize information have generated a heterogeneity of products over the years. Because they are made using various methods and tools, these products are generally not interoperable. Thanks to the ability to share both syntax and semantics information, the use of ontologies and standards is the best way for knowledge management and information exchange. 
CIDOC-CRM (Conceptual Reference Model) (Le Boeuf et al., 2018) can be considered the conceptual ontological reference model. It was developed to manage the cataloguing of the documentation, then other specific ontologies were progressively introduced in order to specify other aspects of the conservation process. The main role of CRM is to allow the exchange of information and the integration among heterogeneous sources. CRM does not define terminology but intends to provide a model of the logical structure aimed at semantic interoperability. The contents of the CRM are standardized by ISO 21127: 2006 (ISO 21127:2006). Offering the conceptual basis for information exchange among cultural heritage institutions is the main purpose of these international standards.

In Italy, the aspects concerning the digital management of building information processes are defined in UNI 11337. (UNI 11337) Some references to cultural heritage are present in the fourth part. However, this part needs to be rewired according to the Conservation process as defined in art. 29 of Cultural Heritage and Landscape Code.

\subsection{Using ontologies for Cultural Heritage}

About the Architectural Cultural Heritage, in recent years research has proposed some examples of the use of ontologies for knowledge and management. Their application for Architectural Cultural Heritage comes much later than the industrialized construction sector. Unfortunately, this time lag is not new.

Some of them are presented here:

Acierno, Cursi, Simeone and Fiorani, in Rome, propose an informative model aimed at representing and managing knowledge for the conservation process by means of a case study. The model is defined by ontology-based representation of the artefact and of the knowledge collected. The information derives and is shared by the various multidisciplinary actors involved during investigation and conservation activities. The project considered as a starting point the ontologies defined by CIDOC-CRM. Due to a new framework that matches the logic structure of the conservation process some additions on the missing aspects has been done. (Acierno et al., 2016)

Cacciotti, Blasco and Valach, Prague, propose an ontological model capable of representing the complexity of degradation phenomena. The acquisition of information derives from diagnostic campaign. The model distinguishes among triggering events, mechanisms, agents and damages. It replicates the basic relations among these factors and their significance in function of possible interventions. It enables the representation of looping damaging process, taking into consideration single or combined processes with simultaneous or delayed occurrences. (Cacciotti et al., 2014)

Messaoudi, Veron, Halin and De Luca, in Marseille, propose an ontology model for semantic annotations aimed at detecting and collecting data concerning the state of conservation. The information comes from photographic survey and point clouds. The ontologies used are: CIDOC-CRM (which provides the common and extensible semantic framework), CRMsci for scientific observation, CRMdig for the origin of data and CRMinf for the argumentation process. (Massaoudi et al., 2017) The topic of ontologies and point clouds for the recognition of degradation phenomena was also presented in the article by Nespeca and De Luca. (Nespeca and De Luca, 2016)
The last example is related to the case of Basilica of Collemaggio in L'Aquila, restored after the 2009 earthquake. The project was developed by Politecnico di Milano, La Sapienza University of Rome, University of L'Aquila, "Soprintendenza Beni Architettonici e Paesaggio" of L'Aquila. It is not an example of the use ontologies to create an informative model, but it is presented in contrast to what has been explained so far because from this experience the limitation of using BIM on Cultural Heritage are clearly showed. The use of the BIM was however necessary and essential to be able to manage the complexity of the entire process (from the survey, through the design phases, to the construction site and execution of the works) and to guarantee interoperability with the other software used. The project showed that architectural BIM, programmed for the construction industry, are not adapted to the cultural heritage due to the high level of standardization of the elements. In this case, other modelling software was used to overcome the "rigidity" of the BIM. (Oreni et al., 2014)

From the bibliographic research, several experiences of compiling ontologies for the $\mathrm{CH}$ have emerged. Others are present in addition to those selected and synthesized. The origin is varied, both at European and global level. A focus on a limited and specific part emerges from the contributions. So, in some cases, the organization and systematization of knowledge is the goal, in others the ontologies are used to describe the conditions of conservation and degradation, even the risk assessment related to the effects of climate change has been highlighted. In all cases there is a lack of the parts relating to the conservation and management process (as defined by the Cultural Heritage and Landscape Code), for example preventive activities, maintenance activities, planning, Facility Management, and so on. Of course, concepts of conservation, valorisation and management defined by the Code have value and meaning limited to the Italian territory. It is equally true that the approach to the conservation of cultural heritage is strongly conditioned by the culture, history, availability and type of heritage, by the legislation that is specific to heritage and general and so on. For this reason, it is difficult to find homogeneous and shared objectives, if not impossible. However, there is no global approach and multi-vision to the whole cultural system, also excluding territorial particularities. Because of this specification of the analysed aspect, the recognition of the multidisciplinary and the interaction of the subjects is also missing. Only a few, in limited passages, consider this fundamental aspect. Experiences have shown a widespread interest in the method and its potential. For these reasons they are however a positive sign, despite the lacks noted

\section{DEVELOPMENT PRESPECTIVES}

The conservation process is clearly defined from a theoretical and regulatory point of view. However, the tools to implement it effectively are still lacking. Aligning the cultural heritage sector with technological evolution is a pressing and urgent need, especially if we consider the mandatary nature of the DM $560 / 2017$. Indeed, the entry into force of the law has caught the sector lacking the necessary tools and therefore unprepared to give an answer.

Starting from the recognition of the need for more representative models, as stated by Rothenberg, it is necessary to identify the models that together represent reality as best as 
possible. The informative models can be BIM, GIS or other information systems such as those for Facility Management or for Conservation Plan. The only necessary condition is that they must be programmed to guarantee semantic interoperability. Interoperability must therefore be multi-systemic.

The various information models should describe the aspects relating to site, history, architecture, structures, system, construction site/security. The site model is represented by a territorial information system. The descriptive contents have to provide the information relating to type, morphology, value (cultural value, according to the Cultural Heritage and Landscape Code), risk (seismic, hydrogeological and environmental, in the mean as the presence of unfavourable conditions for the material conservation), urban planning (on the various levels of scale). This kind of model allows the definition of both prevention and design activities. The historical model should contain the information attributes related to the historical-cultural value. This model is linked to the architectural model of which it is an integral and substantial part. The architectural model describes the classes of elements that make up the building. The classes in the ontologies and their graphic modelling must describe the objects of the historical architecture, with their specific shape and material characterizations. The structure model contains the attributes related to the static aspect, refer to heterogeneity of materials, techniques, construction phases and so on. The systems model can be considered a single model or broken-down various aspects (water-sanitary, electrical, mechanical, and so on). The site construction/security model should describe the attributes related to prevention, maintenance and restoration activities that require interventions with means and works.

It is important to underline that all informative models, in a condition of full interoperability, contribute to the implementation of management process that consist of Conservation, Valorisation and Facility Management activities. So, other information systems as Conservation Plan and Facility Management, also allows long-term planning in order to guarantee the economic sustainability of the process. Ontologies so defined is linked, as already mentioned, to the construction of standards, corresponding to IFC for constructions, but specific for Cultural Heritage.

Interchange platform, fundamental in a condition of multidisciplinary, will aim to constitute a common data environment within it will be possible to guarantee the integration of data, real-time updating and semantic interoperability. With the platform an integrated process will put in place. Creating relationships among the subjects involved is the function of the platform, in order that all aspects are developed coherently with the cultural value of the building.

\section{CONCLUSION}

The contribution highlighted the advantages of the use of computer ontologies for the realization of informative models for Cultural Heritage. For this purpose, the contents that should be present are indicated. The importance of specific foundation classes for Cultural Heritage functional to the semantic interoperability of the models is emphasized. Finally, the need for an information exchange platform is made explicit.

The presented contents are first reflections and steps about informative models' contents, ontologies classes, standard and platform definition. The next activities of CHERIE project and my $\mathrm{Ph} . \mathrm{D}$. research will make them concrete and applicable.

\section{REFERENCES}

Acierno M., Cursi S., Simeone D., Fiorani D., 2017. Architectural heritage knowledge modelling: an ontology-based framework for conservation process. Journal of Cultural Heritage 24, 124-133. 10.1016/j.culher.2016.09.010.

Bittner T., Donnelly M., Winter S., 2006. Ontology and Semantic Interoperability. 10.1201/9781420036282.pt3.

Cacciotti R., Blasko M., Valach J., 2014. A diagnostic ontological model for damages to historical constructions. Journal of Cultural Heritage 16. 40-48. 10.1016/j.culher.2014.02.002.

Le Boeuf P., Doerr M., Ore C. E., Stead S., 2018. CIDOCConceptual Reference Model, Definition. version 6.2.3

Della Torre S., Mirarchi C., Pavan A., 2017. Il BIM per la conservazione, Rappresentare e gestire la conoscenza. ANANKE 82

Gholami E., Kiviniemi A., Sharples S., 2015. Implementing Building Information Modelling (BIM) in Energy-Efficient Domestic Retrofit: Quality Checking of BIM Model. Vol: 32rd international CIB W78 conference

Gruber T.R., 1992. A translation approach to portable ontology specification. Knowledge System Labortory, Technical Report KSL. 92-71

ISO 10303-11:2004 - Industrial automation systems and integration - Product data representation and exchange - Part 11: Description methods: The EXPRESS language reference manual

ISO 16739-1:2018 - Industry Foundation Classes (IFC) for data sharing in the construction and facility management industries - Part 1: Data schema

ISO 21127:2006 - Information and documentation - A reference ontology for the interchange of cultural heritage information

Khan S., Safyan M., 2014. Semantic matching in hierarchical ontologies. Journal of King Saud University - Computer and Information Sciences, 26. 247-257.

10.1016/j.jksuci.2014.03.010.

Kupcík M., Šír M., Bradác Z, 2012. Interoperability through ontologies. Programmable Devices and Embedded Systems. 196-200. 10.3182/20120523-3-CZ-3015.00039.

Laakso M., Kiviniemi A., 2012. The IFC Standard - A Review of History, Development, and Standardization. Journal of Information Technology in Construction, 17

Lee J., Lee Y., Min K., Kim Ju., Kim Ja., 2008. Building ontology to implement the BIM (Building Information Modeling) focused on pre-design stage. The $25^{\text {th }}$ International Symposium on Automation and Robotics in Construction. 2629. 10.22260/ISARC2008/0053. 
Martinez-Cruz C., Blanco I. J., Vila M. A., 2011. Ontologies versus relational databases: are they so different? A comparison. Artificial Intelligence Review 38. 10.1007/s10462-011-9251-9

Messaoudi T., Véron P., Halin G., De Luca L., 2017. An ontological model for the reality-based 3D annotation of heritage building conservation state. Journal of Cultural Heritage, 29. 100-112. 10.1016/j.culher.2017.05.017.

Nespeca, R., De Luca, L., 2016. Analysis, thematic maps and data mining from point cloud to ontology for software development. ISPRS - International Archives of the Photogrammetry, Remote Sensing and Spatial Information Sciences. XLI-B5. 347-354. 10.5194/isprsarchives-XLI-B5347-2016.

Noy N. F., McGuinness D. L., 2001. Ontology Development 101: A Guide to Creating Your First Ontology. Knowledge Systems Laboratory, 32.

Obrst, L., 2003. Ontologies for semantically interoperable systems. International Conference on Information and Knowledge Management, Proceedings. 366-369.

$10.1145 / 956863.956932$.

Oreni D., Brumana R., Della Torre S., Banfi F., Barazetti L., Previtali M., 2014. Survey turned into HBIM: The restoration and the work involved concerning the Basilica di Collemaggio after the earthquake (L'Aquila). ISPRS Annals of Photogrammetry, Remote Sensing and Spatial Information Sciences. II-5. 267-273. 10.5194/isprsannals-II-5-267-2014.

Razniewski, S., Savkovic, O., Nutt, W. (2016). Turning The Partial-Closed World Assumption Upside Down. AMW.

Reiter, R., 1977. On Closed World Data Bases. 55-76. 10.1007/978-1-4684-3384-5_3.

Rothenberg, J., 1989. The nature of modeling. AI, Simulation \& Modeling. 75-92.

Sir M., Bradac Z., Fiedler P., 2015. Ontology versus Database. IFAC-PapersOnLine, 48. 220-225.

10.1016/j.ifacol.2015.07.036.

UNI 11337-1:2017 - Edilizia e opere di ingegneria civile Gestione digitale dei processi in-formativi delle costruzioni Parte 1: Modelli, elaborati e oggetti informativi per prodotti e processi

UNI 11337-4:2017 - Edilizia e opere di ingegneria civile Gestione digitale dei processi in-formativi delle costruzioni Parte 4: Evoluzione e sviluppo informativo di modelli, elaborati e oggetti 